\title{
Albumin-to-Fibrinogen Ratio as an Independent Prognostic Parameter in Untreated Chronic Lymphocytic Leukemia: A Retrospective Study of 191 Cases
}

\author{
Yi-Xin Zou, MD',2,3 \\ Jia Qiao, MD 1,2,3 \\ Hua-Yuan Zhu, MD, PhD',2,3 \\ Rui-Nan Lu, MD, PhD $1,2,3$ \\ Yi Xia, MD, PhD',2,3 \\ Lei Cao, MD'1,3, \\ Wei $\mathbf{W u}, \mathrm{MD}, \mathrm{PhD}^{1,2,3}$ \\ Hui Jin, $\mathrm{PhD}^{1,23}$ \\ Wen-Jie Liu, MD, PhD',2,3 \\ Jin-Hua Liang, MD',2,3 \\ Jia-Zhu Wu, MD, PhD ${ }^{1,23}$ \\ Li Wang, MD 1,2,3 \\ Lei Fan, MD, PhD ${ }^{1,2,3}$ \\ Wei Xu, MD, PhD ${ }^{1,23}$ \\ Jian-Yong Li, MD, $P h D^{1,23}$
}

\begin{abstract}
${ }^{1}$ Department of Hematology, The First Affiliated Hospital of Nanjing Medical University, Jiangsu Province Hospital, Nanjing, ${ }^{2}$ Key Laboratory of Hematology of Nanjing Medical University, Nanjing, ${ }^{3}$ Collaborative Innovation Center for Cancer Personalized Medicine, Nanjing, China
\end{abstract}

Correspondence: Jian-Yong Li, MD, PhD Department of Hematology, The First Affiliated Hospital of Nanjing Medical University, Jiangsu Province Hospital, Nanjing 210029, China Tel: 86-25-83781120

Fax: 86-25-83781120

E-mail: lijianyonglm@126.com

Co-correspondence: Wei $\mathrm{Xu}, \mathrm{MD}, \mathrm{PhD}$

Department of Hematology, The First Affiliated Hospital of Nanjing Medical University, Jiangsu Province Hospital, Nanjing 210029, China Tel: $86-25-83781120$

Fax: 86-25-83781120

E-mail: xuwei10000@hotmail.com

Received June 14, 2018

Accepted July 31, 2018

Published Online August 1, 2018

*Yi-Xin Zou, Jia Qiao, and Hua-Yuan Zhu contributed equally to this work.

\section{Purpose}

Chronic lymphocytic leukemia (CLL) is one of the most frequent type of B-cell chronic lymphoproliferative disorders and chronic inflammation takes part in the development of CLL. However, there has been no valid immune biomarker to predict the prognosis of untreated CLL patients.

\section{Materials and Methods}

In this retrospective study, we analyzed the clinical correlations and prognostic value of albumin-to-fibrinogen ratio (AFR) detected at diagnosis in 191 CLL patients.

\section{Results}

The cut-off value of AFR was 9.7 calculated by X-tile. Patients who were more than 65 years old were often accompanied by low level of AFR $(p<0.001)$. Survival analysis showed that patients with low level of AFR had shorter overall survival (OS) than patients with high level of AFR ( $p<0.001$ ). Multivariate analysis illustrated that AFR had a negative impact on OS $(p=0.003)$ and was independent of parameters involved in CLL international prognostic index and other prognostic markers such as CD38 and ZAP-70.

\section{Conclusion}

These data provide a comprehensive view of AFR and shows that AFR at diagnosis is an adverse prognostic factor in untreated CLL patients.

\section{Key words}

Serum albumin, Fibrinogen, B-cell chronic lymphocytic leukemia, Prognosis 


\section{Introduction}

Chronic lymphocytic leukemia (CLL), a common type of chronic B-cell lymphoproliferative disorders (B-CLPD), seriously harms human health. To date, the etiology of CLL remains unclear, and hereditary factors as well as physical and chemical factors may contribute to the development and progression of this disease. Previous studies demonstrated that chronic inflammation was an enabling characteristic of cancer, and unexceptionally, it played an important role during the course of CLL [1-3].

Serum albumin, an important protein synthesized in liver, is not only an indicator of nutrition supply, but also a marker of inflammatory state. During the process of inflammation, albumin decomposes rapidly and therefore, low content of serum albumin is associated with poor prognosis in many diseases, such as non-small cell lung cancer (NSCLC), prostate cancer and CLL [4-7]. Like serum albumin, fibrinogen is also a marker of inflammation. Previous studies demonstrated that elevated fibrinogen level represented an inferior overall survival (OS) in esophageal carcinoma, NSCLC, prostate cancer, gallbladder cancer and so on [6,8-10]. Albumin-to-fibrinogen ratio (AFR) is a novel immune biomarker to predict prognosis. Previous research showed that in NSCLC, low level of AFR represented an adverse OS, and both time-dependent receiver operating characteristic curve and multivariate Cox regression analysis indicated AFR was a superior prognostic marker when comparing with serum albumin and fibrinogen [11].

So far, there have been a lot of biomarkers related to molecular biology and cytogenetics, such as tumor protein 53 (TP53) and immunoglobulin heavy variable-region gene $(I G H V)$ mutation status, to predict survival of CLL patients. However, immune indices which can effectively evaluate the prognosis of CLL patients remain absent. The prognostic value of AFR in CLL was not reported previously. Whether AFR has the same value to predict survival in CLL as in NSCLC needs further verification. We retrospectively investigated the correlations of AFR with other clinical factors, and examined the impact of AFR level on survival in 191 untreated CLL individuals in order to evaluate whether AFR level could independently predict prognosis.

\section{Materials and Methods}

\section{Patients}

Totally 191 previously untreated CLL patients diagnosed from June 1995 to November 2017 in our hospital were enrolled in this single-center retrospective study. Diagnosis of CLL was based on the International Workshop on CLLNational Cancer Institute criteria.

\section{Data collection}

Laboratorial data such as absolute lymphocyte count, absolute platelet count, hemoglobin, $\beta 2$-microglobulin ( $\beta 2$ $\mathrm{MG})$, serum albumin and fibrinogen were accessible from the hospital-based laboratory service within 24 hours after first admission.

Detection of TP53 mutation and IGHV mutation status were performed as previously described [12]. A germline homology of $98 \%$ as a cut-off value was used to differentiate IGHV mutated from unmutated cases.

Fluorescence in situ hybridization was carried out to detect TP53 deletion according to the procedures described previously [13]. CD38 and ZAP-70 were detected via flow cytometry, and the cut-off value for positivity were $30 \%$ and $20 \%$, respectively.

\section{Statistical analyses}

Cut-off value was determined by use of X-tile [14], a software that allows the user to move a cursor across the grid to test multiple divisions and accept the best $\mathrm{p}$-value. SPSS ver. 23 (IBM Corp., Armonk, NY) was used to analyze data. OS was defined as time from diagnosis to death or last followup and treatment-free survival (TFS) was calculated as time between diagnosis and first-line treatment. Survival curves were constructed by Kaplan-Meier method and log-rank test was used for statistic associations. Comparisons of AFR as continuous parameter in different groups were described using unpaired t test for unpaired samples. The Cox proportional hazards model was established to evaluate factors (including AFR level) at diagnosis on survival by univariate and multivariate analyses. For the multivariate analysis, we included variables whose $p$-value is less than 0.05 during the univariate analysis. $\mathrm{p}<0.05 \mathrm{w}$ as defined as statistical significance.

\section{Ethical statement}

The study was approved by the Ethics Committee of the First Affiliated Hospital of Nanjing Medical University. Sub- 
Table 1. Baseline characteristics of 191 untreated chronic lymphocytic leukemia patients

\begin{tabular}{|c|c|c|c|}
\hline Parameter & No. of cases $(\%)$ & AFR (mean $\pm S D)$ & p-value \\
\hline \multicolumn{4}{|l|}{ Age (yr) } \\
\hline$>65$ & $66(34.6)$ & $15.01 \pm 5.20$ & $<0.001$ \\
\hline$\leq 65$ & $125(65.4)$ & $18.86 \pm 7.82$ & \\
\hline \multicolumn{4}{|l|}{ Sex } \\
\hline Male & $128(67.0)$ & $17.22 \pm 6.04$ & 0.399 \\
\hline Female & $63(33.0)$ & $18.16 \pm 9.25$ & \\
\hline \multicolumn{4}{|l|}{ Binet stage } \\
\hline A & $76(39.8)$ & $17.46 \pm 5.55$ & 0.913 \\
\hline B or C & $115(60.2)$ & $17.58 \pm 8.21$ & \\
\hline \multicolumn{4}{|c|}{ CD38 (>30\%) } \\
\hline Positive & $41(21.5)$ & $17.93 \pm 5.47$ & 0.692 \\
\hline Negative & $150(78.5)$ & $17.42 \pm 7.68$ & \\
\hline \multicolumn{4}{|c|}{ ZAP-70 (> 20\%) } \\
\hline Positive & $86(45.0)$ & $17.87 \pm 5.49$ & 0.555 \\
\hline Negative & $105(55.0)$ & $17.25 \pm 8.44$ & \\
\hline \multicolumn{4}{|c|}{$\beta 2-\mathrm{MG}(\mathrm{mg} / \mathrm{L})$} \\
\hline$>3.5$ & $82(42.9)$ & $16.40 \pm 6.17$ & 0.061 \\
\hline$\leq 3.5$ & $109(57.1)$ & $18.38 \pm 7.89$ & \\
\hline \multicolumn{4}{|c|}{ TP53 disruption } \\
\hline Yes & $43(22.5)$ & $15.74 \pm 5.14$ & 0.066 \\
\hline No & $148(77.5)$ & $18.05 \pm 7.69$ & \\
\hline \multicolumn{4}{|c|}{ IGHV mutation } \\
\hline Yes & $114(59.7)$ & $16.84 \pm 5.54$ & 0.112 \\
\hline No & 77 (40.3) & $18.54 \pm 9.17$ & \\
\hline
\end{tabular}

AFR, albumin-to-fibrinogen ratio; SD, standard deviation; $\beta 2-\mathrm{MG}, \beta 2$-microglobulin; TP53, tumor protein 53; IGHV, immunoglobulin heavy variable-region.

jects provided written informed consent and carried out according to the Declaration of Helsinki.

\section{Results}

\section{Patient characteristics}

Totally 191 previously untreated CLL patients were involved in our study, and their baseline characteristics were showed in Table 1. Median age was 61 years old (range, 23 to 86 years) and the ratio of male/ female was 2.0. There were 76 patients in Binet A stage (39.8\%), and 115 patients in Binet $B$ or $C$ stage $(60.2 \%)$. The number of patients with TP53 disruption and unmutated IGHV were $43(22.5 \%)$ and $77(40.3 \%)$, respectively. Median follow-up was 51 months (range, 1 to 270 months). Totally 124 patients $(64.9 \%)$ received treatment including immunochemotherapy $(109 / 124,87.9 \%)$, ibrutinib $(4 / 124,3.2 \%)$, and data not available $(11 / 124,8.9 \%)$.
At diagnosis, median value of albumin was $42 \mathrm{~g} / \mathrm{L}$ (range, 21.0 to $53.7 \mathrm{~g} / \mathrm{L}$ ), and median value of fibrinogen was 2.49 $\mathrm{g} / \mathrm{L}$ (range, 0.56 to $12.05 \mathrm{~g} / \mathrm{L}$ ). Median value of AFR at diagnosis was 17.19 (range, 2.85 to $79.46 \mathrm{~g} / \mathrm{L}$ ), and the cut-off point of AFR according to the analysis of X-tile yielded the highest difference in OS was 9.70. The numbers of patients whose AFR $\leq 9.70$ (low level) and $>9.70$ (high level) were 20 and 171 , respectively.

\section{Correlations between the AFR value and the other fac- tors}

Correlations between the AFR value and the other factors were presented in Table 1. Patients who were more than 65 years old had lower AFR than young patients $(\mathrm{p}<0.001)$.

\section{Prognostic impact of AFR and clinical outcome}

The OS of patients with low AFR was inferior to that of patients with high AFR ( $<0.001)$ (Fig. 1B). The 3- and 5-year OS was $56.1 \%$ and $56.1 \%$ in low AFR group vs. $92.8 \%$ 
A
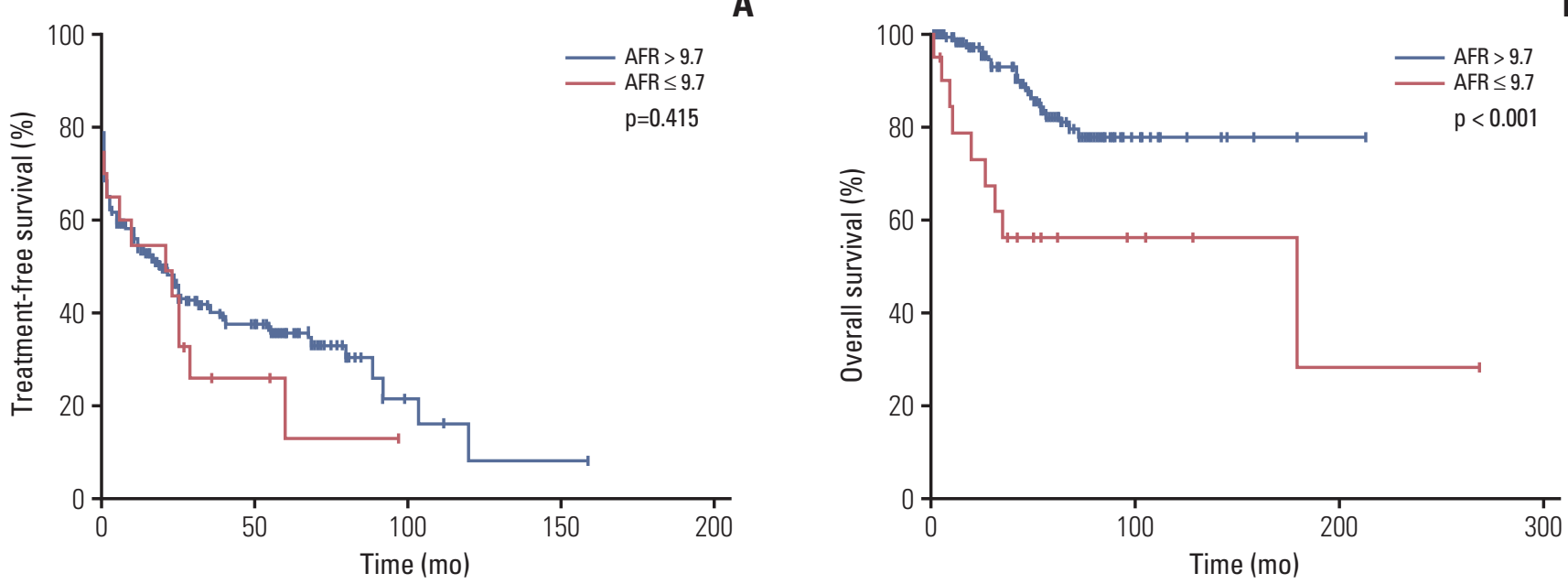

Fig. 1. Kaplan-Meier curves of treatment-free survival (A) and overall survival (B) for different levels of albumin-to-fibrinogen ratio (AFR).

Table 2. Univariable and multivariate analysis of treatment-free survival in 191 patients with chronic lymphocytic leukemia

\begin{tabular}{|c|c|c|c|c|}
\hline \multirow{2}{*}{ Characteristic } & \multicolumn{2}{|c|}{ Univariate analysis } & \multicolumn{2}{|c|}{ Multivariate analysis } \\
\hline & HR $(95 \% \mathrm{CI})$ & p-value & HR $(95 \%$ CI) & p-value \\
\hline Age $>65$ yr & $1.089(0.757-1.568)$ & 0.645 & ND & ND \\
\hline Binet $\geq$ stage $B$ & $2.747(1.836-4.110)$ & $<0.001^{\text {a) }}$ & $2.012(1.302-3.109)$ & $0.002^{\mathrm{a})}$ \\
\hline$\beta 2-\mathrm{MG}>3.5 \mathrm{mg} / \mathrm{L}$ & 2.734 (1.904-3.927) & $<0.001^{\mathrm{a})}$ & $2.023(1.367-2.993)$ & $<0.001^{\text {a) }}$ \\
\hline CD38 $(>30 \%)$ & $1.507(1.003-2.262)$ & $0.048^{\mathrm{a})}$ & $1.274(0.837-1.939)$ & 0.259 \\
\hline ZAP-70 (> 20\%) & $1.168(0.819-1.668)$ & 0.391 & ND & ND \\
\hline TP53 disruption & $1.633(1.079-2.471)$ & $0.020^{\mathrm{a})}$ & $1.237(0.799-1.916)$ & 0.339 \\
\hline IGHV unmutated & 1.439 (1.009-2.052) & $0.045^{\mathrm{a})}$ & $1.038(0.709-1.519)$ & 0.848 \\
\hline $\mathrm{AFR} \leq 9.7$ & $1.237(0.720-2.126)$ & 0.440 & ND & ND \\
\hline
\end{tabular}

HR, hazard ratio; CI, confidence interval; ND, not done; $\beta 2-\mathrm{MG}, \beta 2$-microglobulin; TP53, tumor protein 53; IGHV, immunoglobulin heavy variable-region; AFR, albumin-to-fibrinogen ratio. ${ }^{\text {a) }}$ Statistically significant.

Table 3. Univariable and multivariate analysis of overall survival in 191 patients with chronic lymphocytic leukemia

\begin{tabular}{|c|c|c|c|c|}
\hline \multirow{2}{*}{ Characteristic } & \multicolumn{2}{|c|}{ Univariate analysis } & \multicolumn{2}{|c|}{ Multivariate analysis } \\
\hline & HR $(95 \%$ CI) & p-value & HR $(95 \%$ CI $)$ & p-value \\
\hline Age $>65 y r$ & $2.589(1.296-5.174)$ & $0.007^{\mathrm{a})}$ & $2.456(1.206-5.000)$ & $0.013^{\mathrm{a})}$ \\
\hline Binet $\geq$ stage $B$ & $2.126(0.981-4.607)$ & 0.056 & ND & ND \\
\hline$\beta 2-\mathrm{MG}>3.5 \mathrm{mg} / \mathrm{L}$ & $2.153(1.070-4.332)$ & $0.032^{a)}$ & $0.835(0.386-1.805)$ & 0.646 \\
\hline CD38 $(>30 \%)$ & $1.003(0.385-2.614)$ & 0.995 & ND & ND \\
\hline ZAP-70 (> 20\%) & $1.400(0.702-2.792)$ & 0.340 & ND & ND \\
\hline TP53 disruption & $4.049(2.013-8.147)$ & $<0.001^{\mathrm{a})}$ & $2.900(1.327-6.338)$ & $0.008^{\text {a) }}$ \\
\hline IGHV unmutated & 3.514 (1.690-7.307) & $0.001^{\mathrm{a})}$ & 2.835 (1.239-6.489) & $0.014^{\text {a) }}$ \\
\hline $\mathrm{AFR} \leq 9.7$ & 3.648 (1.667-7.985) & $0.001^{\mathrm{a})}$ & $3.467(1.536-7.824)$ & $0.003^{\mathrm{a})}$ \\
\hline
\end{tabular}

HR, hazard ratio; CI, confidence interval; ND, not done; $\beta 2-\mathrm{MG}, \beta 2$-microglobulin; TP53, tumor protein 53; IGHV, immunoglobulin heavy variable-region; AFR, albumin-to-fibrinogen ratio. ${ }^{\text {a) }}$ Statistically significant. 

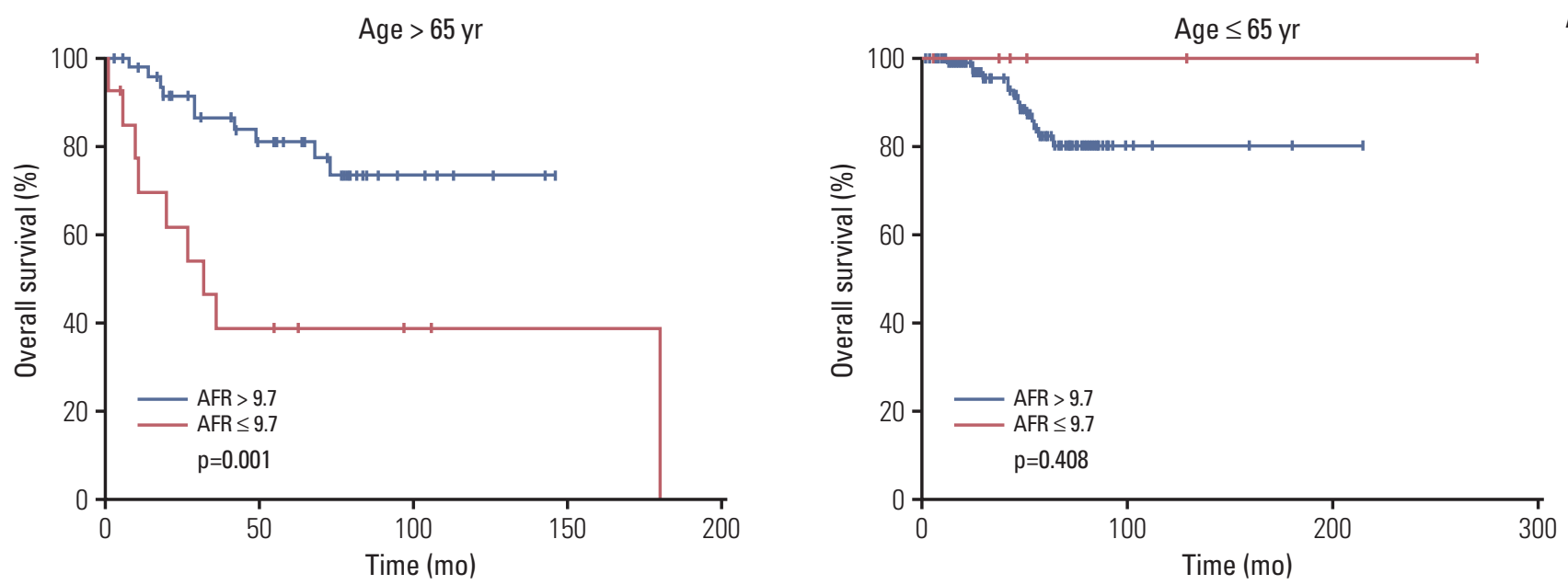

A
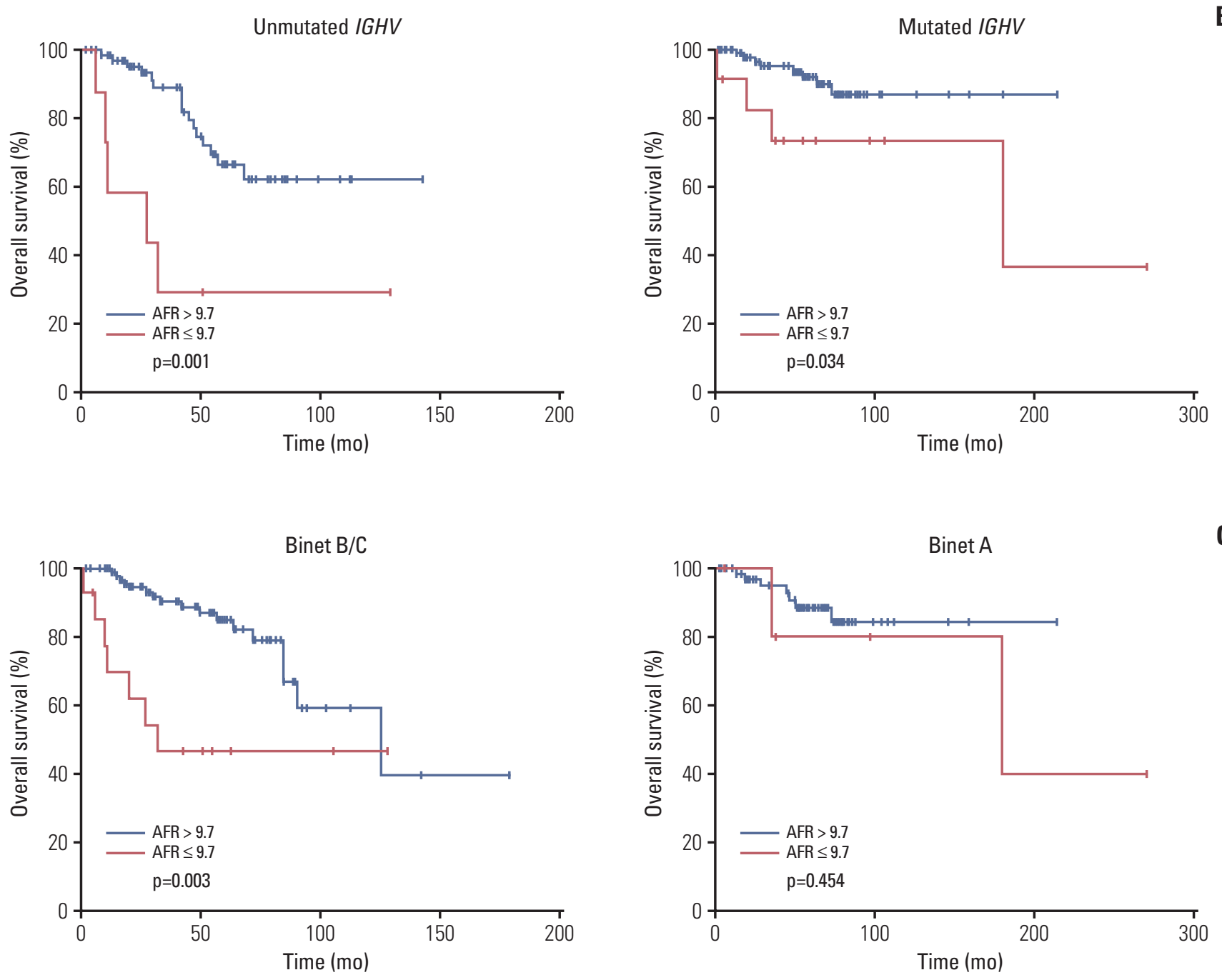

Fig. 2. Kaplan-Meier curves of overall survival for different levels of albumin-to-fibrinogen ratio (AFR) stratified by age (A), immunoglobulin heavy variable-region (IGHV) mutation status (B), Binet stage (C), tumor protein 53 (TP53) status (D), and $\beta 2-$ microglobulin (B2-MG) level (E). (Continued to the next page) 

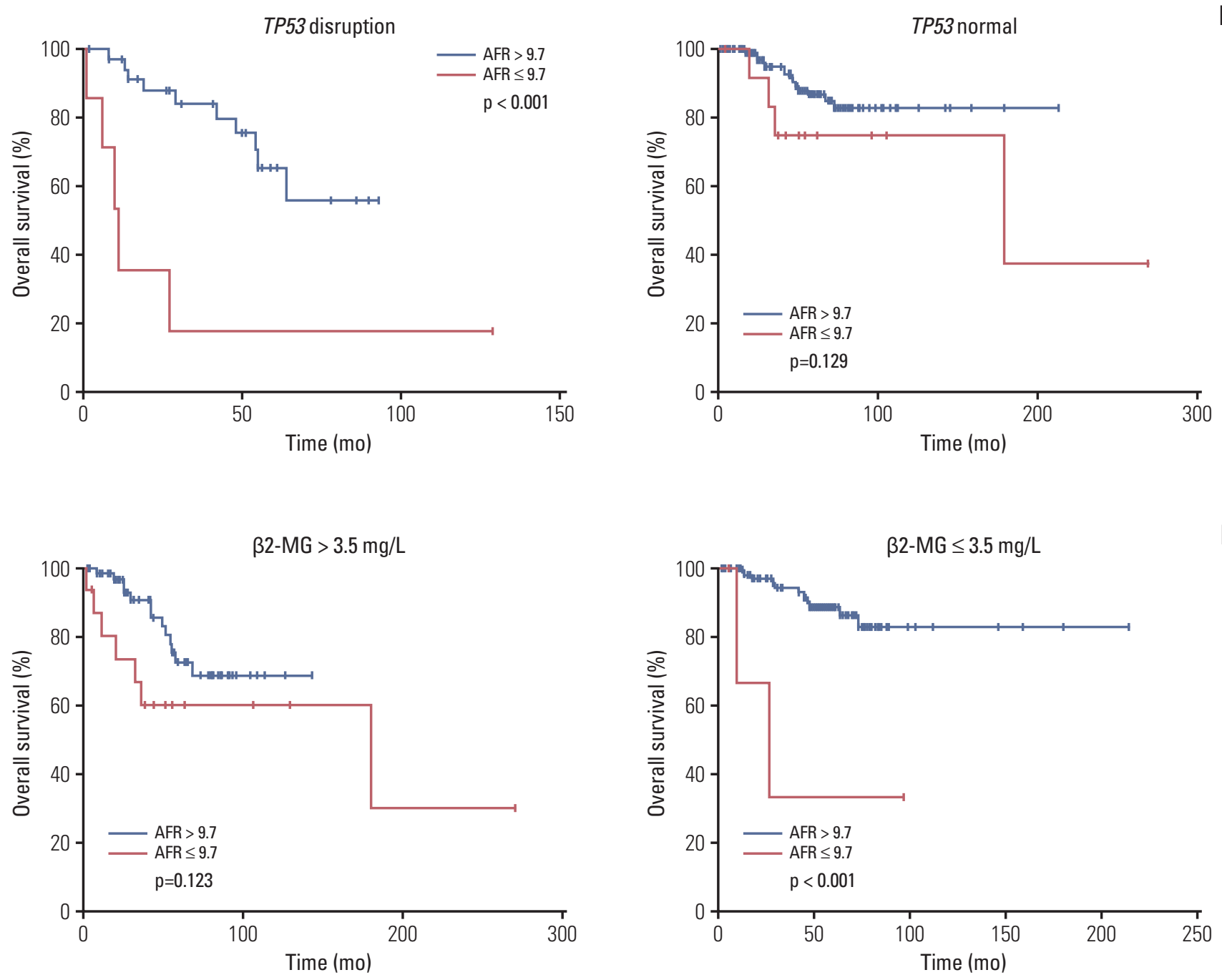

Fig. 2. (Continued from the previous page)

and $82.4 \%$ in high AFR group. However, there was no significant difference of TFS between two groups ( $p=0.415$ ) (Fig. 1A).

Univariate Cox regression analysis showed that Binet $\geq$ stage $\mathrm{B}(\mathrm{p}<0.001), \beta 2-\mathrm{MG}>3.5 \mathrm{mg} / \mathrm{L}(\mathrm{p}<0.001)$, CD38positive $>30 \%(\mathrm{p}=0.048)$, TP53 disruption $(\mathrm{p}=0.020)$, and unmutated IGHV ( $\mathrm{p}=0.045)$ had adverse effects on TFS (Table 2$)$. In addition, age $>65$ years old $(p=0.007), \beta 2-\mathrm{MG}$ $>3.5 \mathrm{mg} / \mathrm{L}(\mathrm{p}=0.032)$, TP53 disruption $(\mathrm{p}<0.001)$, unmutated IGHV $(\mathrm{p}=0.001)$, and AFR $\leq 9.7(\mathrm{p}=0.001)$ were associated with inferior OS (Table 3). Multivariate Cox regression analysis demonstrated that Binet $\geq$ stage $B(p=0.002)$ and $\beta 2-\mathrm{MG}>3.5 \mathrm{mg} / \mathrm{L}(\mathrm{p}<0.001)$ were independent risk factors for TFS and age $>65$ years $(\mathrm{p}=0.013)$, TP53 disruption $(\mathrm{p}=0.008)$, unmutated IGHV ( $\mathrm{p}=0.014)$, and AFR $\leq 9.7(\mathrm{p}=0.003)$ could independently had negative impact on OS.

\section{Subgroup analysis of AFR}

Totally 191 CLL patients were divided into subgroups according to risk factors in CLL international prognostic index (CLL-IPI). Worse OS was seen in individuals with low AFR in subgroups such as age $>65$ years old $(p=0.001)$ (Fig. 2A), unmutated IGHV ( $\mathrm{p}=0.001$ ) (Fig. 2B), mutated IGHV ( $\mathrm{p}=0.034)$ (Fig. 2B), Binet $\mathrm{B} / \mathrm{C}(\mathrm{p}=0.003)$ (Fig. 2C), TP53 disruption ( $p<0.001$ ) (Fig. 2D), and $\beta 2-\mathrm{MG} \leq 3.5 \mathrm{mg} / \mathrm{L}(\mathrm{p}<$ 0.001) (Fig. 2E). 


\section{Discussion}

CLL is one of the most frequent type of B-CLPD. It is well known that microenvironment plays a significant role in the development of CLL and chronic inflammation is mainly involved in this process. It is known to all that the amount of circulating immune cells such as monocytes can affect survival of CLL patients [15]. Proteins such as albumin and fibrinogen are indictors of chronic inflammation and nutrition, and may be a useful marker for prediction of CLL patients' survival. Previous study revealed that AFR could effectively predict prognosis in NSCLC individuals. However, whether AFR has promising prognostic value in CLL patients remains unclear. In this retrospective study, we analyzed the clinical factors and prognosis of 191 untreated CLL patients with different levels of AFR. Patients who were more than 65 years old had lower AFR than young patients. In addition, although it had no statistic difference, it had a tendency that patients with high concentration of $\beta 2-\mathrm{MG}$ or TP53 disruption frequently had low AFR because $\beta 2-\mathrm{MG}$ was also a sensitive biomarker for inflammation [16] and chronic inflammation can be initiated when tumor suppressor gene TP53 disruption [17]. Besides, the high level of $\beta 2-\mathrm{MG}$ and TP53 disruption were clinically related with the inferior prognosis of patients with CLL, and patients with poor prognosis usually hardly survived due to various clinical factors, such as nutrition, organ function, biosynthesis ability and etc., which were strongly related with the content of albumin and fibrinogen. These might account for the relationship between AFR level and $32-\mathrm{MG}$ level or TP53 disruption.

We found that low AFR was an adverse prognostic biomarker for OS, no matter what other adverse prognostic factors they had, raising the possibility that severe inflammation might accelerate and exacerbate the development of CLL. Subgroup analyses showed significant differences of different levels of AFR for OS in patients with age $>65$ years old, unmutated IGHV, mutated IGHV, Binet B/C, TP53 disruption, and $\beta 2-\mathrm{MG} \leq 3.5 \mathrm{mg} / \mathrm{L}$, which demonstrated that AFR level could optimize the prognostic stratification based on CLL-IPI and should be included in the CLL-IPI prognosis model. Other analyses through different validation sets in different methods, such as prospective observation or interventional studies were strongly recommended in order to validate feasibility of adding AFR level into CLL-IPI. In addition, Cox regression analysis also revealed that low AFR level could predict an inferior OS and was independent of factors involved in CLL-IPI and other prognostic markers such as CD38 and ZAP-70.

Why AFR decreased in CLL might be accounted for the following reasons. Serum interleukin 6 and tumor necrosis factor $\alpha$ were in high level in CLL patients, and these two cytokines were adverse markers in CLL and would downregulate the synthesis of albumin [18-20]. In addition, circulating fibroblast growth factor-2 and vascular endothelial growth factor were elevated in CLL patients, and these two proteins, whose serum concentrations were high in patients with advanced diseases, could promote the synthesis of fibrinogen [21,22]. Additionally, when patients were undernourished, both levels of albumin and fibrinogen would decline. However, when chronic inflammation disturbed patients, albumin was reduced while fibrinogen was elevated. Based on this, AFR might be a better biomarker to predict prognosis than single albumin or fibrinogen because ratio could effectively remove nutritional factors and amplify the effects of inflammation.

In summary, we retrospectively explored clinical features and prognostic value of AFR in 191 untreated CLL patients. Low level of AFR was more common in CLL patients who were more than 65 years old. In addition, low AFR level was associated with poor prognosis regardless of other clinical factors and AFR level was an independent prognostic factor for OS. AFR level could also predict the survival of patients stratified according to the biomarkers in CLL-IPI. Further researches containing a larger population and multicenter data are needed to better understand the roles of AFR level in CLL.

\section{Conflicts of Interest}

Conflict of interest relevant to this article was not reported.

\section{Acknowledgments}

This study was supported by National Natural Science Foundation of China (81370657, 81470328, 81600130, 81770166, 81720108002), Jiangsu Province's Medical Elite Programme (ZDRCA2016022), Project of National Key Clinical Specialty, Jiangsu Provincial Special Program of Medical Science (BL2014086 and BE2017751) and National Science and Technology Major Project (2018ZX09734007). 


\section{References}

1. Hanahan D, Weinberg RA. Hallmarks of cancer: the next generation. Cell. 2011;144:646-74.

2. Caligaris-Cappio F. Inflammation, the microenvironment and chronic lymphocytic leukemia. Haematologica. 2011;96:353-5.

3. Rozovski U, Keating MJ, Estrov Z. Targeting inflammatory pathways in chronic lymphocytic leukemia. Crit Rev Oncol Hematol. 2013;88:655-66.

4. Wierda WG, O'Brien S, Wang X, Faderl S, Ferrajoli A, Do KA, et al. Prognostic nomogram and index for overall survival in previously untreated patients with chronic lymphocytic leukemia. Blood. 2007;109:4679-85.

5. Levis A, Ficara F, Marmont F, De Crescenzo A, Resegotti L. Prognostic significance of serum albumin in chronic lymphocytic leukemia. Haematologica. 1991;76:113-9.

6. Wang Y, Chen W, Hu C, Wen X, Pan J, Xu F, et al. Albumin and fibrinogen combined prognostic grade predicts prognosis of patients with prostate cancer. J Cancer. 2017;8:3992-4001.

7. Miura K, Hamanaka K, Koizumi T, Kitaguchi Y, Terada Y, Nakamura D, et al. Clinical significance of preoperative serum albumin level for prognosis in surgically resected patients with non-small cell lung cancer: comparative study of normal lung, emphysema, and pulmonary fibrosis. Lung Cancer. 2017;111:88-95.

8. Wang H, Zhao J, Zhang M, Han L, Wang M, Xingde L. The combination of plasma fibrinogen and neutrophil lymphocyte ratio (F-NLR) is a predictive factor in patients with resectable non small cell lung cancer. J Cell Physiol. 2018;233:4216-24.

9. Xu WY, Zhang HH, Yang XB, Bai Y, Lin JZ, Long JY, et al. Prognostic significance of combined preoperative fibrinogen and CA199 in gallbladder cancer patients. World J Gastroenterol. 2018;24:1451-63.

10. Lv GY, Yu Y, An L, Sun XD, Sun DW. Preoperative plasma fibrinogen is associated with poor prognosis in esophageal carcinoma: a meta-analysis. Clin Transl Oncol. 2018;20:853-61.

11. Li SQ, Jiang YH, Lin J, Zhang J, Sun F, Gao QF, et al. Albuminto-fibrinogen ratio as a promising biomarker to predict clinical outcome of non-small cell lung cancer individuals. Cancer Med. 2018;7:1221-31.

12. Wang WT, Zhu HY, Wu YJ, Xia Y, Wu JZ, Wu W, et al. Elevated absolute NK cell counts in peripheral blood predict good prognosis in chronic lymphocytic leukemia. J Cancer Res
Clin Oncol. 2018;144:449-57.

13. Xia Y, Fan L, Wang L, Gale RP, Wang M, Tian T, et al. Frequencies of SF3B1, NOTCH1, MYD88, BIRC3 and IGHV mutations and TP53 disruptions in Chinese with chronic lymphocytic leukemia: disparities with Europeans. Oncotarget. 2015;6:5426-34.

14. Camp RL, Dolled-Filhart M, Rimm DL. X-tile: a new bioinformatics tool for biomarker assessment and outcome-based cut-point optimization. Clin Cancer Res. 2004;10:7252-9.

15. Herishanu Y, Kay S, Sarid N, Kohan P, Braunstein R, Rotman $\mathrm{R}$, et al. Absolute monocyte count trichotomizes chronic lymphocytic leukemia into high risk patients with immune dysregulation, disease progression and poor survival. Leuk Res. 2013;37:1222-8.

16. Wu HC, Lee LC, Wang WJ. Associations among serum beta 2 microglobulin, malnutrition, inflammation, and advanced cardiovascular event in patients with chronic kidney disease. J Clin Lab Anal. 2017;31:e22056.

17. Pal S, Bhattacharjee A, Ali A, Mandal NC, Mandal SC, Pal M. Chronic inflammation and cancer: potential chemoprevention through nuclear factor kappa B and p53 mutual antagonism. J Inflamm (Lond). 2014;11:23.

18. Rothschild MA, Oratz M, Schreiber SS. Serum albumin. Hepatology. 1988;8:385-401.

19. Emilie D, Leger-Ravet MB, Devergne O, Raphael M, Peuchmaur M, Coumbaras J, et al. Intratumoral production of IL-6 in B cell chronic lymphocytic leukemia and B lymphomas. Leuk Lymphoma. 1993;11:411-7.

20. Jablonska E, Kiersnowska-Rogowska B, Rogowski F, Parfienczyk A, Puzewska W, Bukin M. TNF family molecules in the serum of patients with B-cell chronic lymphocytic leukemia (B-CLL). Leuk Lymphoma. 2005;46:1307-12.

21. Krejci P, Dvorakova D, Krahulcova E, Pachernik J, Mayer J, Hampl A, et al. FGF-2 abnormalities in B cell chronic lymphocytic and chronic myeloid leukemias. Leukemia. 2001;15:22837.

22. Gora-Tybor J, Blonski JZ, Robak T. Circulating vascular endothelial growth factor (VEGF) and its soluble receptors in patients with chronic lymphocytic leukemia. Eur Cytokine Netw. 2005;16:41-6. 